\title{
Raised international normalised ratio (INR): Is it a cause or an effect of late cardiac tamponade?
}

\author{
P S Wong, W B Pugsley
}

\begin{abstract}
Two cases of late cardiac tamponade after valve replacement surgery are reported: both patients were treated with oral anticoagulants (warfarin) after operation. An erratic response in the international normalised ratio (INR) was found before the diagnosis of late tamponade. It is suggested that this response of the INR may be an early indicator of late cardiac tamponade rather than a cause.
\end{abstract}

(Br Heart J 1992;68:212-3)

Late cardiac tamponade is an infrequent but serious complication after cardiac surgery. The reported incidence is $0.1 \%-6 \% .^{1-4}$ The overall mortality has been quoted as $18 \%{ }^{5}$ Our report presents two patients with late cardiac tamponade who were found to have unforeseen increases in international normalised ratio (INR) while receiving warfarin after valve replacement surgery.

\section{Case reports}

A 63 year old man underwent insertion of a Starr-Edwards valve for severe aortic regurgitation. His postoperative recovery was uneventful. Warfarin treatment was started on the second day after operation. He was later transferred to his referral hospital on the seventh day after operation for further convalescence and was discharged home on the 10th day after stabilisation of his INR (between 2.0 and 3.5 ). He presented to his local hospital on the 26th day after operation with a 48 hour history of general malaise and worsening dyspnoea at rest. Two days before admission his INR had been 6.0 and his anticoagulation clinic reduced the dose of warfarin. The patient said that he had not taken medication other than that prescribed by the hospital. Examination of the patient showed signs of cardiac tamponade. The INR was 5.4 and liver function was mildly deranged. Other haematological and biochemical tests were unremarkable. The INR was partially corrected with a transfusion of fresh frozen plasma. Pericardiocentesis was performed after echocardiography had confirmed a large pericardial effusion. Eight hundred millilitres of heavily blood stained fluid was aspirated. The patient eventually recovered successfully.

A 59 year old woman underwent StarrEdwards mitral valve replacement for mixed mitral valve disease. Her recovery after operation was excellent. Warfarin treatment was started on the day after surgery and she was transferred to her referral hospital on the sixth day after operation. She was discharged home three days later. She presented on the 18th day after operation with symptoms and signs of cardiac tamponade. There had been no change in her medication before admission except for a reduction in the dose of the warfarin as instructed by the anticoagulation clinic three days earlier. Her INR had then been 4.6 and despite a reduced warfarin dose was 9.0 on admission. This was partially corrected with a transfusion of fresh frozen plasma. A large pericardial effusion was confirmed by echocardiography, and pericardiocentesis was performed. Nine hundred millilitres of heavily blood stained fluid was aspirated. This resulted in considerable improvement of her haemodynamic state. She then recovered uneventfully.

\section{Discussion}

Late cardiac tamponade was first reported in 1968 by Prewitt et al. ${ }^{3}$ It is defined as cardiac tamponade occurring seven days or more after operation. Most patients reported had had valvar surgery and nearly all were on anticoagulation treatment. ${ }^{24-8}$

The cause of late pericardial effusions is undoubtedly multifactorial. There is always a question of whether the anticoagulants could have triggered the start of late mediastinal haemorrhage, as most patients were receiving anticoagulants when cardiac tamponade occurred. Although no definite relation exists between anticoagulation and the occurrence of delayed cardiac tamponade, it has been suggested that excessive anticoagulation is a principal cause. ${ }^{67}$ It is also possible that the retained mediastinal clot undergoes lysis with subsequent hydrophilic expansion causing cardiac compression.

In both the cases there was an erratic response of the INR to warfarin. Bashi et al and Hardesty et al have reported similar findings. ${ }^{4}$ Bashi et al stated that patients with late cardiac tamponade maintained a high INR irrespective of whether they received a lower dose of warfarin or none at all. ${ }^{4}$ The abnormal results of hepatic function tests, as evident in both our cases, suggest that this unpredictable response to warfarin reflects hepatic dysfunction, possibly as a result of cardiac compression. 
Any patient may take a drug that interacts with warfarin causing a rise in INR. Despite repeated warnings, patients occasionally take other drugs, even aspirin, without telling or consulting their doctors. In our two cases, however, there was no evidence of this.

Increased awareness and recognition of this insidious, but treatable complication should lower the high mortality associated with it. An unexpected prolongation of the INR response may be an early indicator of impending cardiac tamponade. We therefore suggest that this complication be considered whenever there is an erratic response to warfarin.

1 Ofori-Krakye SK, Tyberg TI, Geha AS, Hammond GL Cohen LS, Langou RA. Late cardiac tamponade after open heart surgery: Incidence, role of anticoagulants in its pathogenesis and its relationship to the post-pericardotomy syndrome. Circulation 1981;61:1323-8.

2 Scott RAP, Drew CE. Delayed pericardial effusion with tamponade after cardiac surgery. Br Heart $J$ 1973;35: 1304-6.

3 Prewitt TA, Rackley CE, Wilcox BR, Scatliff JH, Young DT. Cardiac tamponade as a late complication of open heart Cardiac tamponade as a late complication of open heart

4 Bashi VV, Ravikumar E, John S. Delayed tamponade following open heart surgery: Experience with 11 patients. Indian Heart $J$ 1987;39:206-11.

5 Garcia JM, Reyes E, Cheanvechai C, Effler DB. Delayed cardiac tamponade following open-heart surgery. Cleveland Clinic Quarterly 1974;41:103-8.

6 Erdman S, Salomon J, Levy JM. Haemopericardium as a late complication in anticoagulant therapy following mitral valve replacement. Scand J Thorac Cardiovasc Surg 1976;10:205-7.

7 Roos J, Van Joost HE. The cause of bleeding during anticoagulant treatment. Acta Medica Scandinavica 1965; 178:129-31.

8 Hardesty RL, Thompson $M$, Lerberg DB, Siewers RD, O'Toole JD, Salerni R, Bahnson HT. Delayed postoperative cardiac tamponade: Diagnosis and Management. Ann Thorac Surg 1978;26:155-64. 